\title{
Measuring Solar Abundances
}

\author{
R. von Steiger*, J.-C. Vial ${ }^{\dagger}$, P. Bochsler**, M. Chaussidon ${ }^{\ddagger}$, C. M. S. Cohen ${ }^{\S}$, \\ B. Fleck ${ }^{\natural}$, V. S. Heber" ${ }^{\|}$H. Holweger ${ }^{\dagger \dagger}$, K. Issautier**, A. J. Lazarus ${ }^{* *}$, \\ K. W. Ogilvie ${ }^{\S \S}$, J. A. Paquette ${ }^{\text {If }}$, D. B. Reisenfeld ${ }^{* * *}$, L. Teriaca ${ }^{\dagger \dagger}$, K. Wilhelm ${ }^{\dagger \neq}$, \\ S. Yusainee ${ }^{\S \S}$, J. M. Laming ${ }^{\text {If斤 }}$ and R. C. Wiens ${ }^{* * *}$ \\ ${ }^{*}$ International Space Science Institute, Hallerstrasse 6, CH-3012 Bern, Switzerland \\ ${ }^{\dagger}$ Institut d'Astrophysique Spatiale, Université Paris XI - C.N.R.S., Bâtiment 121, F-91405 ORSAY Cedex, France \\ ${ }^{*}$ P hysikalisches Institut, University of Bern, Sidlerstr. 5, CH-3012 Bern, Switzerland \\ ${ }^{\ddagger}$ CRPG-CNRS, Vandoeuvre-lès-Nancy, 54501, France \\ ${ }^{\S}$ California Institute of Technology, Pasadena, CA 91125, USA \\ "ESA Space Science Department, NASA/GSFC Mailcode 682.3, Greenbelt, MD 20015, USA \\ "Institute for Isotope Geology and Mineral Resources, ETH Zürich, 8092 Zürich, Switzerland \\ ${ }^{\dagger}$ Inst. für Theoret. Physik und Astrophysik, Universität Kiel, Kiel, 24098, Germany \\ \#IT, Room 37-687, 77 Mass. Ave., Cambridge, MA 02139, USA \\ ${ }^{\S}$ NASA/GSFC, Code 692, Greenbelt, MD 20771, USA \\ II Department of Physics, University of Maryland, College Park, MD 20742, USA \\ ${ }^{* * *}$ Los Alamos National Laboratory, Mail Stop D466, Los Alamos, NM 87545, USA \\ † Osservatorio Astrofisico di Arcetri, Largo Enrico Fermi 5, Firenze 50125, Italy

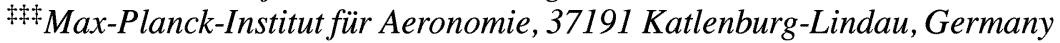 \\ $\S \S$ Univerisiti Teknologi Maralecturer Building, Bandar Jengka, Pahang, 26400, Malaysia

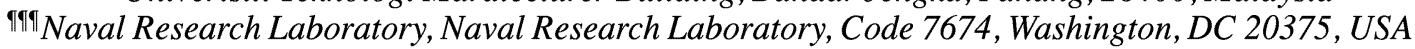

\begin{abstract}
This is the rapporteur paper of Working Group 2 on Measuring Solar Abundances. The working group presented and discussed the different observations and methods for obtaining the elemental and isotopic composition of the Sun, and critically reviewed their results and the accuracies thereof. Furthermore, a few important yet unanswered questions were identified, and the potential of future missions to provide answers was assessed.
\end{abstract}

\section{INTRODUCTION}

This paper is an attempt at summarising the deliberations of Working Group 2 in the Joint SOHO-ACE Workshop on Solar and Galactic Composition. The tasks of this working group were defined by the Scientific Organising Committee, and somewhat extended by the group, to be the following:

- How do we derive solar abundances from remotesensing data?

-What are the major agreements and disagreements?

- What are the advantages and disadvantages of the different methods?

- What are the currently best solar abundances, and how accurate are they?

-What future observations and experiments do we need to improve the situation?
The tasks were addressed in three group meetings during the workshop. First, the group addressed some basic questions, to which the answers caused no controversy. It then went on to present and discuss the various methods with which abundances can be measured in different reservoirs, what elements are accessible to the different methods, how the solar abundances can be inferred from the measurements, and how accurate the resulting values are. Several elemental abundances and isotopic ratios are still poorly known even today, leaving some important questions unanswered. The group identified a few such questions, and finally discussed how future missions will improve our knowledge of solar abundances. The structure of this rapporteur paper follows this outline quite closely. 


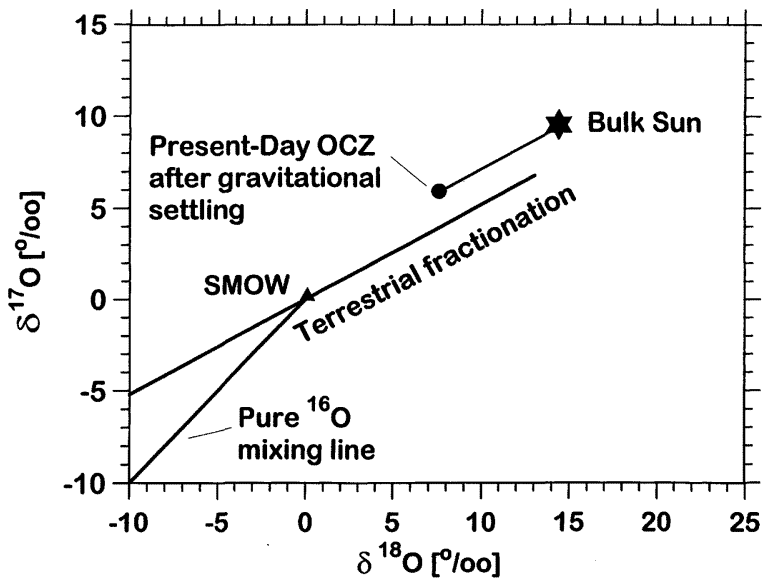

FIGURE 1. Oxygen isotope plot indicating that the heavy isotopes may have been depleted by a few tenths of a percent per mass unit over the life of the Sun [8], adapted from [50]. The axes are labelled in the $\delta$ notation, which indicates the variations of the isotope ratios $\left({ }^{17} \mathrm{O} /{ }^{16} \mathrm{O} v s .{ }^{18} \mathrm{O} /{ }^{16} \mathrm{O}\right)$ relative to a reference value (standard mean ocean water, SMOW) in permil.

\section{What solar abundances?}

The first question is: What are solar abundances, anyway? What is the reservoir that contains them?

Solar abundances are contained in the outer convective zone (OCZ), as represented by the photosphere. Indeed, it is (nearly) pristine, or representative of the protosolar nebula, because it is so large and well-mixed, and because it is sufficiently shallow so as not to reach and dredge up the products of nuclear burning in the core [9]. Nevertheless, a few exceptions have to be kept in mind:

- Deuterium was burnt to form ${ }^{3} \mathrm{He}$ already in the premain sequence phase of the nascent Sun.

- Because of partial mixing below the OCZ, large Li depletion and slight ${ }^{3} \mathrm{He}$ increase can occur.

- ${ }^{4} \mathrm{He}$ has gravitationally settled out of the $\mathrm{OCZ}$ into the top of the radiative zone, an effect of the order of $\approx 10 \%$.

- The same gravitational settling also affects the abundances of heavier elements such as CNO by a few percent, but this change is below the threshold of detectability with today's instrumentation.

- This is not true for the relative abundances of isotopes, though, which are measured with higher accuracy than the elements. Modelling indicates that isotopic abundances can be affected by up to $5 \%$ in the extreme case of ${ }^{3} \mathrm{He} /{ }^{4} \mathrm{He}$ [40], and a few permil per mass unit in the case of heavier elements such as oxygen. Figure 1 (from [8], adapted from [50]) illustrates this for O, which may have shifted from a protosolar value (indicated by a star) along a line with slope $1 / 2$ (as expected for mass-dependent fractionation) to an isotopically lighter value (indicated by a circle).

\section{Why solar abundances?}

The next question is: Why do we need solar abundances? And how accurately do we need to know them?

Solar abundances represent the baseline, or ground truth, for all regimes in the solar system. Only a solid baseline, in particular of the volatiles, allows inferences on how the different regimes were formed and how they evolved over the past $4.6 \mathrm{~Gy}$.

Obviously the baseline must be known more accurately than the natural variability within and between the reservoirs. This variability can be very large, e. g., between the inner and the outer planets, or very small, e. g., in the case of the $\mathrm{O}$ isotopes between Earth, Mars, and asteroids, which is of the order of $10^{-4}$ [50]. Moreover, in the case of natural variability within a reservoir (e. g., the solar wind), the relevant processes causing the fractionations should be understood in sufficient detail so they can be accounted for when deducing the underlying solar abundances.

\section{MEASURING SOLAR ABUNDANCES}

It is not possible, for obvious reasons, to fly a samplereturn mission to the Sun. Consequently, all observations and measurements of solar abundances are indirect. The working group discussed a number of methods for abundance determination and their advantages and disadvantages when deriving solar abundances from them:

- Helioseismology

- optical remote-sensing observations in the solar photosphere (and corona)

- observations of the solar wind, both with in-situ instrumentation and by trapped materials in lunar samples (and meteorites)

- in-situ observations of solar energetic particles

These methods are now discussed in sequence.

\section{Helioseismology}

This method has the advantage to directly probe the OCZ with high accuracy. The determination of solar interior abundances has been recently improved with the help of helioseismology measurements with SOHO. The basic tool is the sound speed variation in the interior 
where the mean molecular weight depends on the composition of the medium. In the OCZ the reference value for helium is $Y=0.249 \pm 0.003$, given by Basu and Antia [6], who used opacities calculated with the OPAL code [36]. Brun et al. [12] concentrated on the discrepancies between "observations" and models at the tachocline (where the turbulent outer region, the convective zone, meets the orderly interior, or radiative zone), and adopting the above-mentioned $Y$ value, and including macroscopic diffusion, they could derive a better determination of the $\mathrm{CNO}$ abundances (found to be underestimated by 2 to $3 \%$ ). According to Turck-Chièze [39], the abundances of heavy elements are determined with 1- $\sigma$ uncertainties of $12 \%(\mathrm{C}), 17 \%(\mathrm{~N}), 17 \%(\mathrm{O}), 15 \%(\mathrm{Ne})$, and $9.5 \%$ (Fe). Overall, the metallicity value quoted in table 3 of $[12]$ is $(2.45 \pm 0.2) \%$.

As far as isotopes are concerned, the ${ }^{3} \mathrm{He} /{ }^{4} \mathrm{He}$ "surface" ratio is increased by $10 \%$ at most. As for the ${ }^{7} \mathrm{Li}$, it seems that its present depletion (by a factor larger than 100) could be accounted for by a small mixing [40].

The results from helioseismology generally give values for the He abundance close to that accepted for standard big bang nucleosynthesis, around $Y=0.25$ [38] or $\mathrm{He} / \mathrm{H}=Y / 4(1-Y)=8.3 \%$. The value of $Y$ usually assumed for the protosolar nebula is a few times 0.01 higher than this, which is also consistent with observations of the He abundance in the gas planets. Uncertainties in helioseismology inversions stem from the approximations made in deriving the equation of state and the opacities used for the solar envelope, but seem unlikely to seriously affect the results (see section 7 and table 14 of [4] for a survey of how the inferred He abundance varies with changes in the solar model, and see [35] for the influence of diffusion mixing). This lowerthan-expected He abundance in the OCZ thus has been taken as evidence of gravitational settling of He.

\section{Optical remote sensing}

This technique has been in use since the 1920s, when Payne-Gaposchkin and Russell first analysed the spectrum of the solar photosphere and extracted the abundances of 56 elements therefrom. Since it is the oldest method it is often taken as the one giving the "standard" solar composition. Yet, like all methods, it has its advantages and disadvantages. The primary advantage is that it directly probes the photosphere, which is representative for the OCZ without any correction. It yields abundances with good accuracy of most elements and even of some isotopes, provided the oscillator strengths are known sufficiently well. On the downside it has to be noted that noble gas abundances, which are particularly important for solar system taxonomy, are inaccessible to the method because these elements have no optical transitions at photospheric temperatures. Moreover, each line stems from one particular ionisation state of an element, so the abundance has to be inferred by dividing by the ionisation fraction of that state at the temperature where it is formed. This is usually done by assuming local thermal equilibrium, which is quite likely a good assumption in the photosphere (but probably less good in the corona - see the report of WG 1 on coronal abundances). Most element abundances are derived from several lines stemming from different charge states, thus reducing the importance of this effect, but they may still be affected as different charge states rarely are observed at the same site in the atmosphere, and as non-Maxwellian distribution functions (e.g., suprathermal tails) may affect the ionisation equilibria.

Photospheric abundances are discussed in detail by Holweger in this volume [24]. Moreover, Patsourakos et $a l$. in this volume [33] show a comparison between observed ion effective temperatures in polar coronal holes (PCH) and model calculations. The overall good agreement between the observations and the model that is based on the ion-cyclotron resonance mechanism of solar wind heating and acceleration, provides new piece of evidence in favour of this mechanism. The above authors also presented some initial theoretical results concerning the iron and oxygen abundances in $\mathrm{PCH}$ within the framework of the ion-cyclotron mechanism. They showed a depletion of iron with respect to oxygen in $\mathrm{PCH}$, a result still to be confirmed.

Optical remote sensing can also be used to pinpoint the sources of the solar wind, such as fast streams emanating from coronal holes: Bright plumes are striking features that, within coronal holes, can extend to more than 30 solar radii from the Sun [e.g., 14]. However, recent analyses of SUMER and UVCS data have shown that the width of UV lines is larger in interplume than in plume regions, hinting to interplumes as the site where energy is preferentially deposited and, possibly, fast wind emanates [e.g., 19, 5, 51].

An important factor that may differentiate plume and interplume regions is their elemental abundance. Ulysses observations have identified a difference in the elemental abundances of the fast and slow wind: low FIP elements are more enriched, with respect to their photospheric abundance, in the slow wind than in the high speed wind. Hence, if interplume regions are really the sources of the fast wind, we may possibly find a different elemental composition between plumes and interplume plasma at coronal levels. Recent measurements of the O VI 1032/1037 $\AA$ line intensity ratio in the interplume lanes are presented by Teriaca et al. in this volume together with an attempt to model the O VI line intensities as a function of height. Their analysis shows that negligible outflow velocities are present below 1.3 solar radii, 
supporting the idea that the fast wind may start being accelerated only above that altitude. They were also able to find a lower limit of 8.5 for the interplume oxygen abundance (on the log scale with $\mathrm{H} \equiv 12$ ). This value is lower than what is measured in the fast solar wind [45] as it could be expected if the oxygen and hydrogen ions move at different speeds at 1.2 solar radii and at about the same speed at $1 \mathrm{AU}$.

\section{In situ solar wind}

The elemental and charge state composition of the solar wind can be observed in situ by space-borne mass spectrometers such as SWICS on Ulysses or CELIAS on SOHO, or using the foil collection technique as on the Apollo missions; the results are summarised in several review papers $[43,8]$. These in situ observations are complementary to the optical remote sensing observations in several ways:

- They comprise a true abundance measurement as all charge states of an element are observed at the same time, so no assumption is needed about thermal equilibrium. The statistical accuracy of the observations can be very high - unless high time resolution is required - but the results are often limited by systematic, instrumental uncertainties, which are very difficult to squeeze below $20 \%$.

- Unlike optical observations in the photosphere, they give good results for the noble gases $\mathrm{He}, \mathrm{Ne}$, and $\mathrm{Ar}$ (and see the next subsection regarding $\mathrm{Kr}$ and $\mathrm{Xe}$ ), in particular the foil collection technique [17].

- The foil collection technique and the newest generation of space mass spectrometers such as CELIASMTOF or ACE-SWIMS can even resolve isotopes of most elements that can be observed, as reviewed in [52].

However, these features come at a price: Due to the relatively small geometric factors of space-borne sensors, or the relatively short exposure time of lunar foils, the observations are still limited to the 10-15 most abundant elements ( $\mathrm{H}, \mathrm{He}, \mathrm{C}, \mathrm{N}, \mathrm{O}, \mathrm{Ne}, \mathrm{Mg}, \mathrm{Si}, \mathrm{S}, \mathrm{Ar}$, and Fe [reviewed in 43], with recent additions of the less abundant $\mathrm{Na}$ [25], $\mathrm{Al}$ [10], $\mathrm{Ca}$ [26, 46], and $\mathrm{Cr}$ [32] from SOHO-CELIAS). More importantly, solar wind abundances are not a genuine, unbiased sample of solar abundances, but they are fractionated. One such fractionation depends on the first ionisation potential (FIP): When comparing solar wind to solar abundances, elements with low FIP $(<10 \mathrm{eV})$ are enriched by a significant factor, the FIP bias, over those with a high FIP. To complicate matters further, the solar wind comes in two quasi-stationary varieties (plus transients such as coronal mass ejections), which differ in strength of the FIP bias: It is about 1.52 and relatively constant in fast streams from coronal holes, while in the slow solar wind from the streamer belt region it is about 2.5-3 on average but highly variable [44] (see also [41] for a brief review of ideas that have been put forward to explain the FIP fractionation). It is tempting to take the fast wind abundances with a modest correction as a good sample of the solar abundances, but care must be taken: If we don't understand the FIP effect and in particular its variability we don't understand solar abundances as derived from the solar wind. Another fractionation process affects mainly helium, causing its abundance in the SW to be only about half of the solar abundance [see, e. g., 1]. It most likely due to insufficient Coulomb drag between protons and alpha particles in the accelerating solar wind.

\section{Trapped solar wind}

Much of the current knowledge on the noble gases in the solar wind is based on the analysis of lunar samples and gas-rich meteorites. The solar wind noble gas data obtained from these "targets" are in good agreement with data obtained from in-situ measurements and the Apollo foils (e.g., for $\mathrm{He}, \mathrm{Ne}$ and $\mathrm{Ar}$ [7, 34, 30]), which gives us confidence to make predictions also for $\mathrm{Kr}$ and $\mathrm{Xe}$. Because of the long exposure time of the meteoritic and lunar samples, their analysis is so far the only method to obtain solar wind $\mathrm{Kr}$ and $\mathrm{Xe}$ abundances as well as the $\mathrm{Kr}$ and $\mathrm{Xe}$ isotopic composition. Precisions on the order of $1 \%$ for elemental ratios and a few permil for isotopic ratios are possible. The solar wind composition deduced from these extraterrestrial dust samples is very important to investigate the solar history, because some of them collected their noble gases several billion years ago.

However, the noble gas record in lunar and meteoritic regolith samples is not straightforward to read. Diffusive loss or redistribution of light noble gases within the grains may influence the original solar wind composition. Experiments on mineral separates have shown that the most retentive $\mathrm{Fe}-\mathrm{Ni}$ grains conserve the true relative abundances of solar $\mathrm{He}, \mathrm{Ne}, \mathrm{Ar}[34,30]$, but $\mathrm{Fe}-$ $\mathrm{Ni}$ is very rare in lunar samples. Ilmenite, another retentive mineral phase, which is abundant in lunar samples, has lost some of its solar $\mathrm{He}$ and $\mathrm{Ne}$, but apparently without altering the isotopic composition of these gases [7]. A further difficulty arises when one compares samples that contain solar wind of different antiquities. Alteration processes on the Moon and meteoritic parent bodies may remove part of the solar wind containing grain surfaces probably in a time-dependent manner. Furthermore, other noble gas components can compro- 
mise the solar wind signal, e. g., cosmogenic noble gases due to long exposure to galactic cosmic rays, or primordial noble gases (especially $\mathrm{Kr}$ and $\mathrm{Xe}$ ) in chondrites. With the help of the in-vacuo-etch-technique (closed system stepped etching in a device directly connected to a mass spectrometer [37]) these components can at least partly be separated from each other. This is done by dissolving only the uppermost solar wind bearing grain layers, thus avoiding e.g., cosmogenic contributions from deeper layers. Furthermore, this release technique avoids noble gas fractionation in the laboratory, because the gases are released at room temperature by slowly etching the carrier minerals and not by diffusion as is the case for stepwise heating.

Now two examples show how solar wind noble gases in extraterrestrial dust samples are important to investigate the solar history. The ${ }^{3} \mathrm{He} /{ }^{4} \mathrm{He}$ ratio in the solar wind and its long time evolution are of great interest, because this ratio is a very sensitive indicator for admixing of material of interior layers into the outer convective zone [9]. New data, obtained by very high-resolution in-vacuo etching of lunar grains of different solar wind antiquity show at face value an enrichment of ${ }^{3} \mathrm{He}$ of around 5\%/Gy. However, even this small apparent increase can probably be explained by secondary processes on the Moon [23].

Lunar soil samples appear to be very well suited to study the isotopic composition of solar wind $\mathrm{Kr}$ and $\mathrm{Xe}$ because the moon is extremely depleted in indigenous $\mathrm{Kr}$ and Xe. Wieler et al. [47, 48, 49] showed variable $\mathrm{Xe}$ and $\mathrm{Kr}$ enrichments in the solar wind, compared to bulk solar composition [3]. The enrichment factor for $\mathrm{Xe}$ is similar to that observed for low-FIP elements in the slow solar wind [43], whereas $\mathrm{Kr}$ is less enriched. At least some regolithic meteorites probably collected solar wind from the early Sun. Analysis of these samples therefore enlarges the data base. Unfortunately there is so far no method to determine the solar wind antiquity in meteorites as is possible for lunar samples. But the data available so far suggest that the FIP-related enrichment of $\mathrm{Xe}$ and $\mathrm{Kr}$ has been even more variable in the past than it was known from lunar samples and - if at least some of the meteorites were irradiated very early - the FIP effect was already active in the early Sun, as further discussed by Heber et al. in this volume.

\section{Solar energetic particles}

Solar energetic particle (SEP) events are transient occurrences which are related to coronal mass ejections (CMEs) and flares on the Sun. The particles are typically accelerated out of the corona and solar wind by CME-driven shocks. They can also be accelerated at the site of flares and propagate outward to be observed at 1 AU. Current instrumentation for measuring SEPs is very mature and enables low fluxes of particles to be measured. The mass resolution of current measurements is good enough to resolve many isotopes of heavy ions between $\mathrm{C}$ and $\mathrm{Ni}$. Because of such excellent instrumentation, the relative abundances of elements and isotopes in SEP events as observed at $1 \mathrm{AU}$ can be quite accurately determined, not only averaged over a single SEP event but often as a function of time within an event.

It has been observed that the composition of SEP events can vary substantially from event to event [28]. In comparing these variations to an average composition, they can often be organised by the charge to mass ratios of the elements [11]. Such fractionation is probably an acceleration and/or transport effect. An additional fractionation, with respect to the photospheric composition (as determined from optical measurements), governed by the first ionisation potential (FIP) of an element is also apparent in the SEP composition [13]. This is the same type of fractionation that is so often observed in the solar wind and like the solar wind, the degree of fractionation varies. Only by correcting for these fractionations a measurement of the photospheric abundances can be obtained using SEP measurements for both elements and isotopes. The abundances of SEPs are therefore better suited to accurately probing acceleration processes and plasma effects rather than to inferring the bulk solar composition.

\section{Summary}

So, what are the best solar abundances that are currently available? Of course, that depends on the element, and as we have seen above there is no single method which gives all abundances with good accuracy. Rather, the abundances must be compiled from a large number of publications, and the values given must be used with scrutiny. Fortunately, several review papers exist doing just that: The classical paper of Anders and Grevesse [3] with two updates [21, 22], or the table in LandoltBörnstein [31]. These tables have the advantage of being complete and readily available, and as such [3] has found very widespread use as a reference, in particular in the astronomical literature. This is of course ok if it is understood that the table lists reference, not necessarily solar abundances, as several values have been updated in the meantime.

The tables generally show very good agreement between solar and meteoritic abundances, which allows to infer gaps in the solar record from the meteoritic one (and vice versa). However, this immediately brings the danger of using circular arguments. For example, noble gases 
can be obtained by interpolating between s-process elements in the meteoritic record, or by inferring from the solar wind record. If the two methods give results which are in agreement we are tempted to attribute them a high degree of credibility, but they could still both be wrong.

Another point worth noting is the evolution of the oxygen abundance over the past decade: 8.93 on the scale where $H \equiv 12$ ) in [3], 8.83 in [21], and 8.73 in [22], while the newest original paper even gives $8.69 \pm 0.05$ [2]. This decrease is of course not a solar effect, but reflects our improving knowledge and interpretation of the relevant lines in the solar spectrum (see also the paper of Holweger in this volume [24]).

In Table 1 we have summarised our conservative estimates of the relative accuracies that can be expected when deriving solar abundances from the different reservoirs discussed above.

\section{SOME UNANSWERED QUESTIONS}

The Working Group has identified a number of unanswered questions (UQs), the answers to which have great potential to advancing our knowledge of the Sun and the solar system. These are:

UQ1: What are the solar isotope ratios, in particular of $N, O$, and $M g$ ? The isotopes are the most convincing tool for taxonomy in the solar system. In the isotope system of oxygen, relative differences of the order of $10^{-4}$ are used to infer the parent of meteorites. Yet the solar ratios are known no better than to $15 \%$.

UQ2: What are the solar noble gas abundances? The noble gases, owing to their high volatility, are another important means for taxonomy in the solar system. Yet all we have are solar wind, not solar abundances. Even a relative knowledge of noble gas abundances among themselves would be helpful, but this only leads to the next question:

UQ3: Is the solar wind a faithful sample of the outer convective zone? Do we understand the FIP effect well enough to infer solar abundances? Although the FIP effect is not expected to cause substantial mass fractionation $[42,29]$, it may still be significant. Specifically, the cause of the added variability of the slow solar wind remains unknown (but see [16]).

UQ4: What causes the contradictory evidence between in-situ charge state and optical observations? Observations of solar wind charge states can be interpreted as a proxy for the coronal electron temperature at the site where the solar wind is formed. Results of UlyssesSWICS in the fast solar wind indicate a coronal hole temperature profile with a maximum of $\approx 1.5 \mathrm{MK}$ at an altitude of $\approx 1.5 R_{\odot}[18,27]$. On the other hand, SOHOSUMER observations above the limb in coronal holes in-

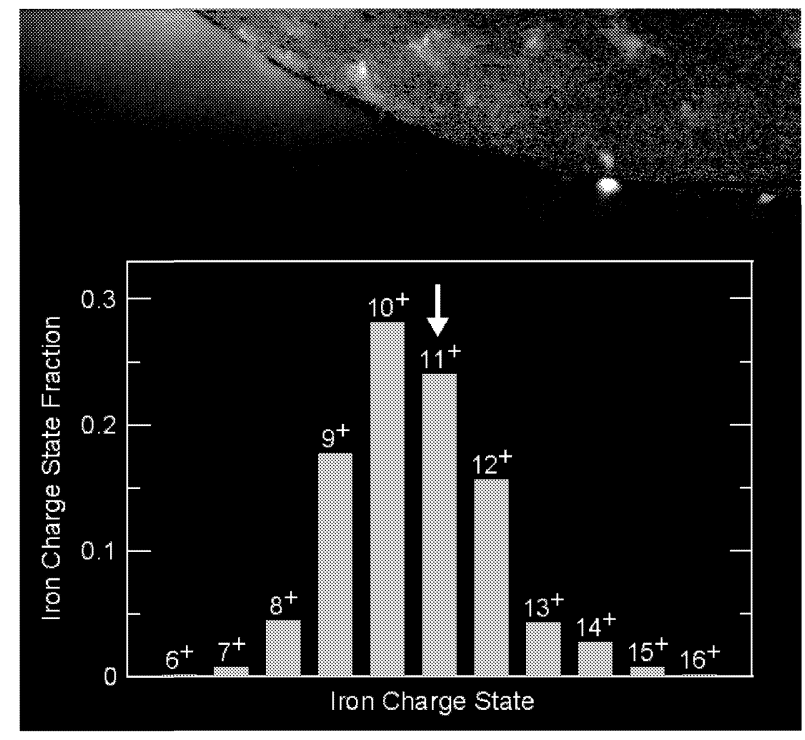

FIGURE 2. SOHO-SUMER picture in the Fe XII line, showing essentially no emission from the coronal hole, and UlyssesSWICS Fe charge state spectrum in the fast solar wind, indicating a large fraction of the corresponding ion $\mathrm{Fe}^{11+}$ (arrow).

dicate electron temperatures barely reaching $1 \mathrm{MK}$ near the limb and decreasing with altitude [51]. The discrepancy is made particularly apparent when looking at the charge states of iron: Whereas SUMER pictures in the $195-\AA$ line of Fe XII are essentially black, SWICS observes a substantial fraction of this ion, $\mathrm{Fe}^{11+} / \mathrm{Fe} \simeq 25 \%$ (see Figure 2). The cause of this discrepancy is as yet unknown. It may equally well be rooted in the interpretation of optical observations, specifically in the assumption of ionisation equilibrium for the charge state fractions, or in the interpretation of charge state spectra observed in situ, which may be affected by differential streaming between ion species and/or by suprathermal tails of the electron distribution function when evolving over the first few solar radii [15], or both.

\section{FUTURE OBSERVATIONS}

In the near future our knowledge about solar abundances will undoubtedly be much improved by the Genesis mission, which is specifically designed to determine elemental and isotopic abundances with the highest feasible accuracy. Other missions will also carry in situ composition instrumentation, notably the Stereo, Solar Orbiter, and Solar Probe missions. In contrast to the Genesis mission, these missions are not specifically designed to measure the solar wind composition per se. Rather, the objective of these missions is to use the abundance measurements as a diagnostic tool to gain further insight into the ori- 
TABLE 1. Table of the relative accuracy of solar abundances as obtained from different reservoirs.

\begin{tabular}{|c|c|c|c|c|c|c|}
\hline Element & Interior & OCZ & Photosphere & Corona & Solar wind & SEP \\
\hline $\mathrm{H}$ & & & $0.3 \%$ & $\lesssim 5 \%$ & $\approx 3 \%$ & \\
\hline $\mathrm{He}$ & $1 \%$ & $1 \%$ & - & $10 \%$ & $\lesssim 10 \%$ & $5 \%$ \\
\hline $\mathrm{Li}$ & & & $15 \%$ & & & \\
\hline CNO etc. & & & $10 \%$ & $\lesssim 50 \%$ & $\lesssim 30 \%$ & $10 \%$ \\
\hline Nobles & & & - & $\lesssim 50 \%$ & $\lesssim 30 \% *$ & $10 \%$ \\
\hline Trans-Fe & & & $10 \%$ & $\lesssim 50 \%$ & & $\gtrsim 100 \%$ \\
\hline Isotopes & & & $15 \%^{\dagger}$ & - & $\approx 10 \%$ & $\approx 15 \%$ \\
\hline
\end{tabular}

gin of the solar wind, in particular its acceleration and fractionation processes.

\section{Genesis}

Genesis is a 3-year NASA mission to capture an integrated sample of solar wind and return it to Earth for ground-based isotopic and elemental analysis in the style of the Apollo-era Solar Wind Composition 'sunshade' experiments. After its launch on 8 August, 2001, the spacecraft spends $\approx 2.5$ years at the L1 point collecting solar wind before returning with a capsule-style reentry in September, 2004. A number of factors should lead to greatly expanded analytical capabilities relative to the Apollo foil collection experiments. These include much longer collection time ( $>2$ years vs. 2 days), absence of lunar dust contamination, and higher purity collection materials, including $\mathrm{Si}, \mathrm{Ge}$, and $\mathrm{SiC}$ wafers, $\mathrm{Au}-$ and Al-on-sapphire, and CVD diamond. The Genesis payload also includes a solar wind concentrator aimed at obtaining $20 \times$ higher-concentration samples of elements lighter than $\mathrm{Si}$, designed particularly for oxygen isotopes. Current technology suggests that abundances will be obtainable for many elements up to and including rare-earth elements. With these capabilities in mind, the science team laid out a large number of prioritised solar wind measurement objectives. The highest priorities are isotope ratios, including ${ }^{16,17,18} \mathrm{O}$ to $\pm 0.1 \% 2-\sigma$ uncertainty, nitrogen isotopes to $\pm 1 \%$, carbon isotopes to $0.4 \%$, and noble gas elemental and isotopic ratios to varying uncertainties. Other priorities include checks on solar nebula solid-gas fractionation, heavy-light element comparisons, $\mathrm{Li}, \mathrm{Be}, \mathrm{B}$ measurements, determinations of ${ }^{14} \mathrm{C}$ and ${ }^{10} \mathrm{Be}$, measurement of solar spallation-produced $\mathrm{F}$, and general comparisons with terrestrial, chondritic, and s-process predicted abundances.

The required accuracies and precisions for the highest priority measurements were based on the need to distinguish between competing science claims. For example, for oxygen isotopes, although differences between
Earth, Mars, and Vesta are significantly smaller, the Genesis measurement goal of $\pm 0.1 \%$ will clearly distinguish between competing theories for the phenomenon of oxygen isotope heterogeneity. It should be noted that current solar and solar-wind isotopic measurements have accuracies only at the $\approx 10 \%$ level. The oxygen isotope issue, and the rationales for the various Genesis measurement objectives, are discussed in greater detail in [50] and in the working group papers 4 and 5 elsewhere in this volume.

An obvious drawback is that the Genesis mission measures solar wind compositions, which are one step removed from the actual solar composition. To aid in understanding the corrections needed to obtain accurate solar abundances from solar wind data, the Genesis spacecraft is collecting separate samples of three different solar wind types: interstream, coronal hole, and coronal mass ejection material, selected by real-time on-board analysis of measurements by ion and electron spectrometers. By studying time-integrated samples of these three different solar wind regimes, it is expected that any isotopic fractionation between the photosphere and solar wind will for the first time be well constrained, and that general limits on elemental fractionation will lead to increased accuracy for solar elemental abundances as well.

\section{Stereo}

The principal scientific objective of the Stereo mission is to understand the origin and consequences of coronal mass ejections (CMEs). It will provide a totally new perspective on solar eruptions by moving away from our customary Earth-bound lookout point and providing stereoscopic views of solar eruptions, with one spacecraft leading Earth in its orbit and another lagging it. Each will carry a cluster of telescopes. When simultaneous telescopic images are combined with data from observatories on the ground or in low Earth orbit, the buildup of magnetic energy, and the lift off, and the trajectory of Earthward-bound CMEs can all be tracked in 
three dimensions. When a CME reaches Earth's orbit, magnetometers and plasma sensors on the Stereo spacecraft will sample the material and allow investigators to link the plasmas and magnetic fields unambiguously to their origins on the Sun. Launch is scheduled for December 2005 .

One of the four instruments selected to fly on Stereo is the "Plasma And SupraThermal Ion Composition" (PLASTIC) instrument, which is the primary sensor for studying coronal-solar wind and solar wind-heliospheric processes. The PLASTIC measurements will be complemented at higher energies by the "In-situ Measurements of Particles and CME Transients" (IMPACT) - a suite of seven instruments that will sample the 3-D distribution of solar wind plasma electrons, the characteristics of the solar energetic particle (SEP) ions and electrons, and the local vector magnetic field.

\section{Solar Orbiter}

The key mission objective of the Solar Orbiter is to study the Sun from close-up (45 solar radii, or $0.21 \mathrm{AU}$ ) in an orbit tuned to solar rotation in order to examine the solar surface and the space above from a co-rotating vantage point at high spatial resolution. The Solar Orbiter will also provide images of the Sun's polar regions from heliographic latitudes as high as $38^{\circ}$. The mission was approved by ESA as a flexi-mission, to be implemented in the time frame 2008-2013.

The Solar Orbiter, while taking high-resolution images and making spectroscopic measurements of solar wind source regions that are magnetically linked to the spacecraft location and making simultaneous, in-situ measurements over the long co-rotation intervals, is ideally suited to address the critical issues of the chromospheric fractionation process. Also, the coronagraph on the Solar Orbiter may include a He channel, which would allow to determine for the first time the helium abundance (high FIP element) in those atmospheric layers where the acceleration of the slow and fast streams actually occurs, and where the charge states freeze in. The results expected will provide keys for the understanding of the processes at the origin of the solar wind and of the elemental composition in the heliosphere.

The strawman payload of the Solar Orbiter includes a Solar Wind Plasma Analyser (SWA), the three principal science goals of which are: (i) to provide observational constraints on kinetic plasma properties for a fundamental and detailed theoretical treatment of all aspects of coronal heating; (ii) to investigate charge- and mass-dependent fractionation processes of the solar wind acceleration process in the inner corona; (iii) to correlate comprehensive in-situ plasma analysis and compo- sitional tracer diagnostics with space-based and groundbased optical observations of individual stream elements. Furthermore, the Solar Wind Plasma Analyser on the Solar Orbiter will investigate in detail ${ }^{3} \mathrm{He}$ and unusual charge states in CME-related flows, as well as the "recycling" of solar wind ions on dust grains in the distance range which has been located as the inner source [20]. Freshly produced pick-up ions from this inner source are specially suited as test particles for studying the dynamics of incorporation of these particles into the solar wind.

The SWA will measure separately the three-dimensional distribution functions of the major solar wind constituents: protons, alpha-particles and electrons. The basic moments of the distributions, such as density, velocity, temperature tensor, and heat flux vector will be obtained under all solar wind conditions and be sampled sufficiently rapidly to characterise fully the fluid and kinetic state of the wind. In this way we will be able to determine possible non-gyrotropic features of the distributions, ion beams, temperature anisotropies, and particle signatures of wave excitation and dissipation. In addition, measurements of representative high-FIP elements (the C, O, N group) and of low-FIP elements (such as Fe, $\mathrm{Si}$ or $\mathrm{Mg}$ ) will be carried out in order to obtain their abundances, velocities, temperature anisotropies and charge states, to probe the wave-particle couplings (heavy-ion wave surfing), and to determine the freeze-in temperatures (as a proxy for the coronal electron temperature).

\section{Solar Probe}

Solar Probe is a NASA mission diving into the outer corona and thus will obtain an extreme close-up glimpse of the solar atmosphere. It will carry two complementary instrumentation packages, one for optical remotesensing and another for in situ particle observations. Unfortunately, the selection process for these packages is currently interrupted and the prospects for the mission are unclear.

The optical measurements made at such a close distance of the Sun as 3 solar radii above the surface will allow for the determination of many elements at different ionisation stages and possibly at stages below the freezein temperatures. This unique feature could help to understand the discrepancies between in-situ freezing-in temperatures and temperatures derived from spectroscopic measurements, as discussed above. Open and closed regions will be explored as well. Table 1 of the Announcement of Opportunity provides a description of the quantities to be measured in-situ: (i) Spectra of energetic particles of $\mathrm{H},{ }^{3} \mathrm{He},{ }^{4} \mathrm{He}, \mathrm{C}, \mathrm{O}, \mathrm{Si}, \mathrm{Fe}$ with a sensitivity of $10 \mathrm{~cm}^{-2} \mathrm{~s}^{-1} \mathrm{sr}^{-1} \mathrm{keV}^{-1}$, a dynamic range of $10^{7}$, and a time resolution of a few seconds in the spectral range 
of $0.02-2 \mathrm{MeV} /$ nucleon. (ii) Time averaged distribution functions of $\mathrm{H}^{+},{ }^{3} \mathrm{He}^{++},{ }^{4} \mathrm{He}^{++}, \mathrm{C}^{n+}, \mathrm{O}^{n+}, \mathrm{Si}^{n+}$, and $\mathrm{Fe}^{n+}$ with a sensitivity of $10 \mathrm{~km} / \mathrm{s}$, a time resolution of $1 \mathrm{~s}\left(\mathrm{H}, \mathrm{He}, \mathrm{e}^{-}\right)$to $10 \mathrm{~s}$ (ions), a dynamic range of $2 \times 10^{7}$, in the spectral range of $0.05-10 \mathrm{keV} /$ charge. It is envisaged to even have nadir view!

As far as remote sensing is concerned, there is no spectroscopic capability but a better understanding of the small-scale properties of the solar wind and, possibly, a determination of the acceleration process would help to understand the fractionation processes occurring in both the slow and fast solar wind.

\section{CONCLUSIONS}

We have seen that solar abundances can be measured using several different methods, any of which has its advantages and disadvantages. The accuracy of the results is reasonably good (depending on the specific question addressed), and future missions will improve on them significantly. Still, there remains the ultimate question: Is there any way to obtain true solar abundances? Most likely the answer is no: Neither with today's technology nor in the foreseeable future will it be possible to fly a mission to the Sun, obtain a sample of the OCZ, and return it to the laboratory for high-precision analysis of all its constituents. Our efforts should rather go into a different direction: As all observations of solar abundances, remote-sensing or in-situ, are affected by physical processes that may or may not alter those abundances, it is of utmost importance to identify all such processes and work towards a detailed, quantitative understanding of their effects. Such understanding can only be obtained and advanced through collaborations of observers, experimentalists, modelers, and theorists, which is exactly the goal of workshops such as this one ... and future ones of this kind.

\section{ACKNOWLEDGMENTS}

We gratefully acknowledge the effort of the scientific and local organisation committees, in particular of Robert F. Wimmer-Schweingruber, in making this workshop happen. We also thank Sylvie Vauclair and Robert F. Wimmer-Schweingruber for useful comments on the manuscript. JML was supported by NRL/ONR Solar Magnetism and the Earth's Environment 6.1 Research Option and by NASA Contracts W19473 and S137836.

\section{REFERENCES}

1. Aellig, M. R., Lazarus, A. J., and Steinberg, J. T., Geophys. Res. Lett. 28, (2001) 2767-2770.

2. Allende Prieto, C., Lambert, D. L., and Asplund, M., Astrophys. J. Lett. 556, (2001) L63-L66.

3. Anders, E., and Grevesse, N., Geochim. Cosmochim. Acta 53, (1989) 197-214.

4. Bahcall, J. N., Pinsonneault, M. H., and Basu, S., Astrophys. J. 555, (2001) 990-1012.

5. Banerjee, D., Teriaca, L., Doyle, J. G., and Lemaire, P., Sol. Phys. 194, (2000) 43-58.

6. Basu, S., and Antia, H. M., Mon. Not. R. Astr. Soc. 276, (1995) 1402-1408.

7. Benkert, J.-P., Baur, H., Signer, P., and Wieler, R., J. Geophys. Res. 98, (1993) 13,147-13,162.

8. Bochsler, P., Reviews of Geophysics 38, (2000) 247-266.

9. Bochsler, P., Geiss, J., and Maeder, A., Sol. Phys. 128, (1990) 203-215.

10. Bochsler, P., Ipavich, F. M., Paquette, J. A., Weygand, J. M., and Wurz, P., J. Geophys. Res. 105, (2000) $12,659-12,666$.

11. Breneman, H. H., and Stone, E. C., Astrophys. J. 299, (1985) L57-L61.

12. Brun, A. S., Turck-Chièze, S., and Zahn, J. P., Astrophys. J. 525, (1999) 1032-1041.

13. Cook, W. R., Stone, E. C., and Vogt, R. E., Astrophys. J. 279, (1984) 827-838.

14. DeForest, C. E., Plunkett, S. P., and Andrews, M. D., Astrophys. J. 546, (2001) 569-575.

15. Esser, R., and Edgar, R. J., Astrophys. J. Lett. 532, (2000) L71-L74.

16. Fisk, L. A., Zurbuchen, T. H., and Schwadron, N. A., Astrophys. J. 521, (1999) 868-877.

17. Geiss, J., Bühler, F., Cerutti, H., Eberhardt, P., and Filleux, C., in Apollo-16 Preliminary Science Report, NASA-SP 315, 1972 pp. 14/1-14/10.

18. Geiss, J., Gloeckler, G., von Steiger, R., Balsiger, H., Fisk, L. A., Galvin, A. B., Ipavich, F. M., Livi, S., McKenzie, J. F., Ogilvie, K. W., and Wilken, B., Science 268, (1995) 1033-1036.

19. Giordano, S., Antonucci, E., Noci, G., Romoli, M., and Kohl, J. L., Astrophys. J. Lett. 531, (2000) L79-L82.

20. Gloeckler, G., and Geiss, J., Space Sci. Rev. 86, (1998) 127-159.

21. Grevesse, N., and Sauval, A. J., Space Sci.Rev. 85, (1998) 161-174.

22. Grevesse, N., and Sauval, A. J., Adv. Space Res. In press.

23. Heber, V. S., Baur, H., and Wieler, R., in 64th Meteoritical Society Conference, Rome, Italy, 2001.

24. Holweger, H., in Solar and Galactic Composition, edited by R. F. Wimmer-Schweingruber, vol. this volume, Woodbury, NY: AIP conference proceedings, 2001.

25. Ipavich, F. M., Bochsler, P., Lasley, S., Paquette, J., and Wurz, P., Eos Trans. AGU 80 (17), (2000) S256.

26. Kern, O., Wimmer-Schweingruber, R. F., Bochsler, P., and Hamilton, D. C., in Correlated Phenomena at the Sun, in the Heliosphere and in Geospace, edited by A. Wilson, vol. 415 of ESA SP, Noordwijk, The Netherlands: ESA Publ. Div., 1997 pp. $345-348$.

27. Ko, Y.-K., Fisk, L. A., Geiss, J., Gloeckler, G., and Guhathakurta, M., Sol. Phys. 171, (1997) 345-361. 
28. Leske, R. A., Mewaldt, R. A., Cohen, C. M. S., Cummings, A. C., Stone, E. C., Wiedenbeck, M. E., Christian, E. R., and von Rosenvinge, T. T., Geophys. Res. Lett. 26, (1999) 2693+.

29. Marsch, E., von Steiger, R., and Bochsler, P., Astron. Astrophys. 301, (1995) 261-276.

30. Murer, C., Baur, H., Signer, P., and Wieler, R., Geochim. Cosmochim. Acta 61, (1997) 1303-1314.

31. Palme, H., and Beer, H., in Landolt-Börnstein - Astronomy and Astrophysics, vol. VI/3A of New Series, Heidelberg: Springer, 1993 pp. 199-222.

32. Paquette, J. A., Ipavich, F. M., Lasley, S. E., Wurz, P., and Bochsler, P., in Solar and Galactic Composition, edited by R. F. Wimmer-Schweingruber, vol. this volume, Woodbury, NY: AIP conference proceedings, 2001.

33. Patsourakos, S., Habbal, S.-R., Vial, J.-C., and Hu, Y. Q., in Solar and Galactic Composition, edited by R. F. Wimmer-Schweingruber, vol. this volume, Woodbury, NY: AIP conference proceedings, 2001 .

34. Pedroni, A., and Begemann, F., Meteoritics 29, (1994) 632-642.

35. Richard, O., Vauclair, S., Charbonnel, C., and Dziembowski, W. A., Astron. Astrophys. 312, (1996) 1000-1011.

36. Rogers, F. J., and Iglesias, C. A., Space Sci. Rev. 85, (1998) 61-70.

37. Signer, P., Baur, H., and Wieler, R., in Alfred O. Nier Symposium on Inorganic Mass Spectrometry, Durango, Colorado, 1993 pp. 181-202.

38. Thuan, T. X., and Izotov, Y. I., Space Sci. Rev. 84, (1998) 83-94.

39. Turck-Chièze, S., Space Sci. Rev. 85, (1998) 125-132.

40. Vauclair, S., Space Sci. Rev. 85, (1998) 71-78.
41. von Steiger, R., Space Sci. Rev. 85, (1998) 407-418.

42. von Steiger, R., and Geiss, J., Astron. Astrophys. 225, (1989) 222-238.

43. von Steiger, R., Geiss, J., and Gloeckler, G., in Cosmic Winds and the Heliosphere, edited by J. R. Jokipii, C. P. Sonett, and M. S. Giampapa, Tucson: The University of Arizona Press, 1997 pp. 581-616.

44. von Steiger, R., Schwadron, N. A., Fisk, L. A., Geiss, J., Gloeckler, G., Hefti, S., Wilken, B., WimmerSchweingruber, R. F., and Zurbuchen, T. H., J. Geophys. Res. 105, (2000) 27,217-27,236.

45. von Steiger, R., Wimmer-Schweingruber, R. F., Geiss, J., and Gloeckler, G., Adv. Space Res. 15, (1995) (7)3-(7)12.

46. Weygand, J., Wurz, P., Bochsler, P., Paquette, J. A., and Ipavich, F. M., in Solar and Galactic Composition, edited by R. F. Wimmer-Schweingruber, vol. this volume, Woodbury, NY: AIP conference proceedings, 2001 .

47. Wieler, R., and Baur, H., Meteoritics 29, (1994) 570-580.

48. Wieler, R., and Baur, H., Astrophys. J. 453, (1995) 987-997.

49. Wieler, R., Kehm, K., Meshik, A. P., and Hohenberg, C. M., Nature 384, (1996) 46-49.

50. Wiens, R. C., Huss, G. R., and Burnett, D. S., Meteoritics and Planetary Science 34, (1999) 99-107.

51. Wilhelm, K., Marsch, E., Dwivedi, B. N., Hassler, D. M., Lemaire, P., Gabriel, A., and Huber, M. C. E., Astrophys. J. 500, (1998) 1023-1038.

52. Wimmer-Schweingruber, R. F., Bochsler, P., and Wurz, P., in Solar Wind Nine, edited by S. R. Habbal, R. Esser, J. V. Hollweg, and P. A. Isenberg, AIP Conference Proceedings, Woodbury, NY: AIP Press, 1999 pp. 147-152. 